\title{
A Network Analysis of the Five Facets Mindfulness Questionnaire (FFMQ)
}

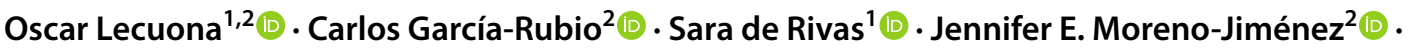 \\ Rosa Marta Meda-Lara ${ }^{3}$ (D) Raquel Rodríguez-Carvajal ${ }^{2}$ (D)
}

Accepted: 11 July 2021

(c) The Author(s) 2021

\begin{abstract}
Objectives The Five Facet Mindfulness Questionnaire (FFMQ) is a popular self-report instrument for mindfulness assessment. However, several studies report mixed evidence regarding its reliability and validity. While recent replication studies have shown several issues regarding its latent structure, first-order facets seemed to replicate successfully. This study proposes an exploratory approach to these facets on an item level in one sample, with cross-validation in another sample.

Methods Using a snowball sampling, 1008 participants were recruited in the first sample. Psychometric networks were applied to explore relations between items and item clusters. We compared these exploratory latent variable proposals with previous literature. A second sample of 1210 participants was collected from an FFMQ validation study, and confirmatory factor analyses were applied to cross-validate findings on the first sample.

Results The FFMQ showed a positively correlated network. Exploratory analyses suggested the 5-facet structure as stable with alternatives of 4-facet (merging Observe and Non-Judging) and 6-facet (splitting Acting with Awareness in two) solutions. However, the CFAs in the second sample did not provide clear support to any solution.

Conclusions The FFMQ showed unclear evidence on its latent structure. We propose researchers and users of the FFMQ to use the most fitting solution between the 5 and 6-facet solutions in their data, since the 4-facet solution is difficult to interpret. We also propose cautionary notes and guidelines for researchers and applied users of the FFMQ and regarding this instrument. We conclude that more research is needed in mindfulness assessment to provide robust measurements.
\end{abstract}

Keywords Mindfulness; FFMQ; Network analysis; Psychometric networks; Exploratory graph analysis; Confirmatory factor analysis

Mindfulness research has experimented a rapid growth in interest and production (American Mindfulness Research Association, 2021). This interest has drawn even political attention (The Mindfulness All-Party Parliamentary Group, 2015), and great importance in academia exploring its positive effects (for a review, see Creswell, 2016). Thus, reliable and valid measurement models and instruments are

Raquel Rodríguez-Carvajal

raquel.rodriguez@uam.es

1 Faculty of Health Sciences, King Juan Carlos University, Móstoles, Spain

2 Department of Biological \& Health Psychology, Faculty of Psychology, Autonomous University of Madrid, Ivan Pavlov St., 6, 28049 Madrid, Spain

3 Health Science University Centre, University of Guadalajara, Guadalajara, Mexico necessary to validate and expand this demand for evidencebased mindfulness research (for a review, see Bergomi et al., 2013b). The Five Facet Mindfulness Questionnaire (FFMQ; Baer et al., 2008) is a well-known and used selfreport instrument to measure mindfulness, since a large body of literature using it.

FFMQ items measure mindfulness as an overall psychological construct with five main facets, named: "Observe," as the tendency to observe stimuli (e.g., sensations of the wind in the body); "Describe," as the tendency to verbally describe those stimuli; "Acting with Awareness" or "Actaware," as the tendency to attend to one's activities in the present moment (in contrast with behaving mechanically, or driven by "automatic pilot"); "Non-Judging of Inner Experience" or "Non-Judging," as the tendency to not judge a particular inner experience as good or bad; "Non-Reacting to Inner Experience" or "Non-Reacting," as the tendency 
to not immediately react to a particular inner experience and "take a step back" to gain perspective. The popularity of the FFMQ is somehow due to a high number of international validations of the questionnaire (Lecuona et al., 2020). Nevertheless, scientific literature has drawn some criticisms toward the FFMQ. For example, evidence suggests that mindfulness facets seem to be stable and valid while the overall mindfulness factor seems unstable. At the same time, the external validity of the instrument remains robust except for the Observe facet in the general population (i.e., nonmeditators). Structuring these comments, criticisms provide challenging evidence on (1) construct, (2) external, and (3) content validity.

As regards (1) construct validity, a recent review and replication study reported several issues (Lecuona et al., 2020). First, most validation studies of the FFMQ cast concerns regarding their data analysis, which questions the apparent robustness of the FFMQ in the literature. Second, the latent structure could not be reproduced as a whole, requiring the inclusion of method factors and the removal of the Observe facet. Therefore, the replication concluded that the FFMQ seems to have an unstable overall mindfulness factor in the general population. However, mindfulness facets (first-level latent factors) seem to replicate successfully.

As regards (2) external validity, a recent meta-analysis (Carpenter et al., 2019) suggests similar conclusions as for construct validity (i.e., facets behaving psychometrically correct except for the Observe facet) and expands them. Concretely, it points out that although the FFMQ seems to be inversely related to negative affect and psychopathological symptoms, the Observe facet is not meaningfully correlated in the same way within general population (i.e., majority of non-meditators). Therefore, the Observe facet seems to not fit well to contribute to the overall mindfulness score, as well as to the external validity of the instrument.

Finally, as regards (3) content validity, a study provided evidence on how Buddhist expert meditators have little agreement on the dimensions of FFMQ as mindfulness components, and also had a consistent disagreement with the Observe facet (Belzer et al., 2013). Furthermore, another study showed that the FFMQ failed to show discriminant validity between a mindfulness-based intervention (MBI) and active controls (Goldberg et al., 2015).

Therefore, it appears that the FFMQ has mixed evidence in the scientific literature. This state-of-the-art can produce undesirable consequences for the field. Skeptics could oversimplify or exaggerate this criticism (e.g., arguing MBIs hold null effects due to the FFMQ issues). It is also predictable for a large force of applied users to ignore criticisms and use the standard (and apparently not robust) model of the FFMQ. This dissonance between technical and applied research has taken place in other fields, like Cronbach's $\alpha$ misuse (Mcneish, 2017). Therefore, while research develops new scalable instruments to measure mindfulness, an indepth exploration of its properties seems necessary to (1) acknowledge which components of the FFMQ are valid, and (2) offer an intermediate solution for both applied and research uses.

It can be argued that although the FFMQ can be used to assess mindfulness facets, the structure of said facets needs more evidence to be considered solid, especially regarding the Observe facet. The reason is that most of the FFMQ validation studies assessed the hierarchical model (assuming a second-order overall mindfulness factor). In contrast, previous studies did not prioritize the correlated 5 -facet model. Due to this, inter-facet correlations, crossloadings, or correlations between items of different facets were not explored in depth. In this regard, the field requires more exploratory techniques than CFA. Exploratory Factor Analysis (EFA) and Exploratory Structural Equation Models (ESEM) are examples of these techniques. However, a recent technique can explore more freely how items relate to each other, namely the psychometric network models (Epskamp et al., 2018). Also known as Regularized Partial Correlation Networks (RPCNs), they have been recently introduced in psychological research (e.g., transdiagnostic models in psychopathology).

RPCNs allow to freely estimate correlations between psychological variables without being caused by an unobserved latent entity. In addition, they provide more unbiased estimations with a three-step approach: First, they compute the correlation matrix (in the case for Likert variables, polychoric correlations); second, they partialize all correlations for the effect of all other correlations; and third, they regularize them (i.e., forcing all small correlations to zero), assuming them to be non-meaningful and aiming for a parsimonious solution (further reading in Epskamp et al., 2018). Thus, RPCNs stand out as a more flexible technique to explore complex interactions between observed variables. Moreover, RPCNs can be used to estimate stable groups of variables via Exploratory Graph Analysis (EGA, Golino \& Epskamp, 2017). These groups can be interpreted as emergent phenomena that arise from the networks, which is compatible with latent variables. Given EGA's performance has shown to be as precise as standard EFA techniques like parallel analysis (Golino et al., 2020), it seems suitable as an interesting option to explore the FFMQ at a first-order level. However, since RPCNs and EGA are novel techniques, a cross-validation technique should benefit the study. In this sense, confirmatory factor analysis (CFA) seems like the optimal technique since it provides testing for specific solutions, like the outputs produced by the EGA.

The FFMQ seems to provide stable facets (except for Observe in some contexts), but literature has not explored them deeply. Thus, this study explores the FFMQ at a facet level via psychometric networks. More concretely, it 
explores the facet solutions that may underlie the FFMQ via RPCNs and EGA. We expect the five-facet theoretical model to be reproduced in our data, and therefore replicate the facet structure of the FFMQ.

\section{Method}

\section{Participants}

We collected two samples for this study, for exploratory and confirmatory analyses, respectively. The first sample was a snowball sample of 1008 participants. We recruited participants for the first sample using a three-step approach. First, we contacted university students from the Faculties of Psychology of the Autonomous University of Madrid and the King Juan Carlos University Madrid asking for participation. Second, we contacted a mindfulness institute to recruit participants before starting mindfulness-based interventions. Third, we posted on our social media profiles (i.e., Facebook, Twitter, Whatsapp, and Telegram) a call for participants. In all steps, all participants were asked to post on their social media profiles our call for participants and contact their relatives to ask for participation. We conducted a power analysis for the estimated networks extracting three indices: True-estimated network correlation, sensitivity (true positives rate), and specificity (true negatives rate) (Epskamp $\&$ Fried, 2018). For our overall sample, we obtained a high correlation (>0.95) and specificity values (around 88\%), while sensitivity (around 68\%) provided more modest values. However, figures of the power analysis (available at https://osf.io/65abr) show how sensitivity values are close to asymptotic. Since RPCNs are designed to prefer higher specificity than sensitivity, these results are expectable. Participants identified predominantly as women (75.89\%), young $(M=35.84$ years, $\mathrm{SD}=13.31)$, with college studies or above $(60.90 \%)$, and had practiced meditation on at least two occasions $(72.59 \%)$.

The second sample was collected by Meda et al. (2015) as an incidental student sample of 1210 participants. Participants identified predominantly as women $(67.62 \%)$, young $(M=20.10$ years, $\mathrm{SD}=5.15)$, with college studies or above $(90.10 \%)$. Meditation experience was not collected.

\section{Procedure}

All recruited participants signed an informed consent of participation, which informed them about the privacy, anonymity, and confidentiality of the treatment of their data, following the Helsinki protocol. Then, we administered a copy of the FFMQ along with demographic items. All data were collected in Spanish. We explained the rules of responding to the questionnaire. The participants could ask any question or issue that they had to the researcher in charge of the data collection, and they could withdraw from the questionnaire at their convenience. The participants did not receive any compensation for their participation. Finally, the second sample was collected by contacting the researchers in charge of the sample and agreeing on providing the dataset (details are provided in Meda et al., 2015).

\section{Measures}

\section{The Five Facets of Mindfulness Questionnaire}

The FFMQ consists of 39 self-reported items with a Likert response format from 1 (totally disagree) to 5 (totally agree) (Baer et al., 2008; validated to Spanish population by Cebolla et al., 2012). As discussed, it has a proposed latent structure of five facets and a hierarchical or bifactor overall facet with potential method factors, with an alternative structure of six facets (Karl et al., 2020; Lecuona et al., 2020).

\section{Data Analyses}

Descriptive statistics were obtained for all variables and plots were examined. We applied RPCNs (Epskamp et al., 2018) to explore relations among items without assuming any structure or model. RPCNs import the network analysis framework to relations between psychological variables. Thus, network nodes (the basic components of the network) represent variables, and edges (links between nodes) represent partial regularized correlations. Mathematically, RPCNs take a correlation matrix (in this case, the polychoric correlation matrix since all items were ordinal) as an input to partialize all correlations for all the other present correlations in the matrix. Then, it regularizes the false positive rate of the network forcing near-zero correlations to zero, assuming them to be essentially uncorrelated. We chose the Expected Bayesian Inference Criteria (EBIC) estimation with the graphical Least Absolute Shrinkage Optimization (gLASSO) regularization as the network estimation method since it provides the most adaptable estimations for ordinal, approximately normal data. Besides, a proportion of explained variance for each node by the network can be obtained with an $R^{2}$ statistic. The final product of this process is a partial regularized correlation matrix, named as "weight matrix."

Following Mullarkey et al. (2018), we examined item standard deviations with an exclusion criterion of below 2.5 SDs of standard deviations since small-variance items can influence the final estimates of the network. No items displayed standard deviations below the criterion. Also, conceptually or empirically overlapping items can bias network estimations since they could measure latent traits without relevant, unique content. Therefore, we 
searched for empirically overlapped items with a threestep approach: (1) we selected pairs of items with potential conceptual overlap and estimated their correlations, (2) obtained their mean and SD to form a criterion of $1 \mathrm{SD}$ as empirically overlapping items, and (3) created composite scores for each resulting pair, reducing the network. Two pair of items met our criteria. Once the final network was reduced and estimated, we applied bootstrap techniques to the network to assess replicability of the network. Among others, we implemented the correlation stability coefficient (CS) to assess stability of edges, with values above 0.25 as minimum, and values above 0.5 as ideal (Epskamp et al., 2018). Once the replicability of the network is assessed, we proceeded to represent the network graphically (see Fig. 1 for an example). We chose the Fruchterman-Reingold algorithm since it provides visually clear and intuitive displays (all data are available at the Open Science Framework-OSF, in Supplementary Materials: https://osf. io/65abr/).
Once the network is estimated, it is relevant to assess the relative importance of each node. Two analytic concepts can be provided: centrality and clustering. Centrality involves the intensity, closeness and inter-connectivity of each node. Clustering involves how much a node is closely connected to a closed group of other nodes (e.g. items clustered due to a latent variable). Centrality measures will not be considered in this study due to bias and interpretation issues when latent variables are involved (Bringmann et al., 2019).

Nevertheless, clustering was assessed with a five-step approach: First, we marked nodes with colors corresponding to theoretical facets to assess if current networks reproduced the model. Second, since Fruchterman-Reingold plots do not provide interpretable distances between nodes, we implemented multi-dimensional scaling plots (see OSF Supplementary Materials) for better assessment of clusters. Third, we performed an Exploratory Graph Analysis (EGA, Golino \& Epskamp, 2017) to explore latent variables in the network. This technique has shown comparable performance
Fig. 1 Estimated network of the FFMQ with the 5-facet solution-Fruchterman-Reingold method. Note: Items are rounded in circles, with pies representing the explained variance $\left(R^{2}\right)$ of each item. Lines connecting items represent correlations, blue $=$ positive correlation, red $=$ negative correlation. Highly correlated items tend to be closer, while non-correlated nodes tend to be farther

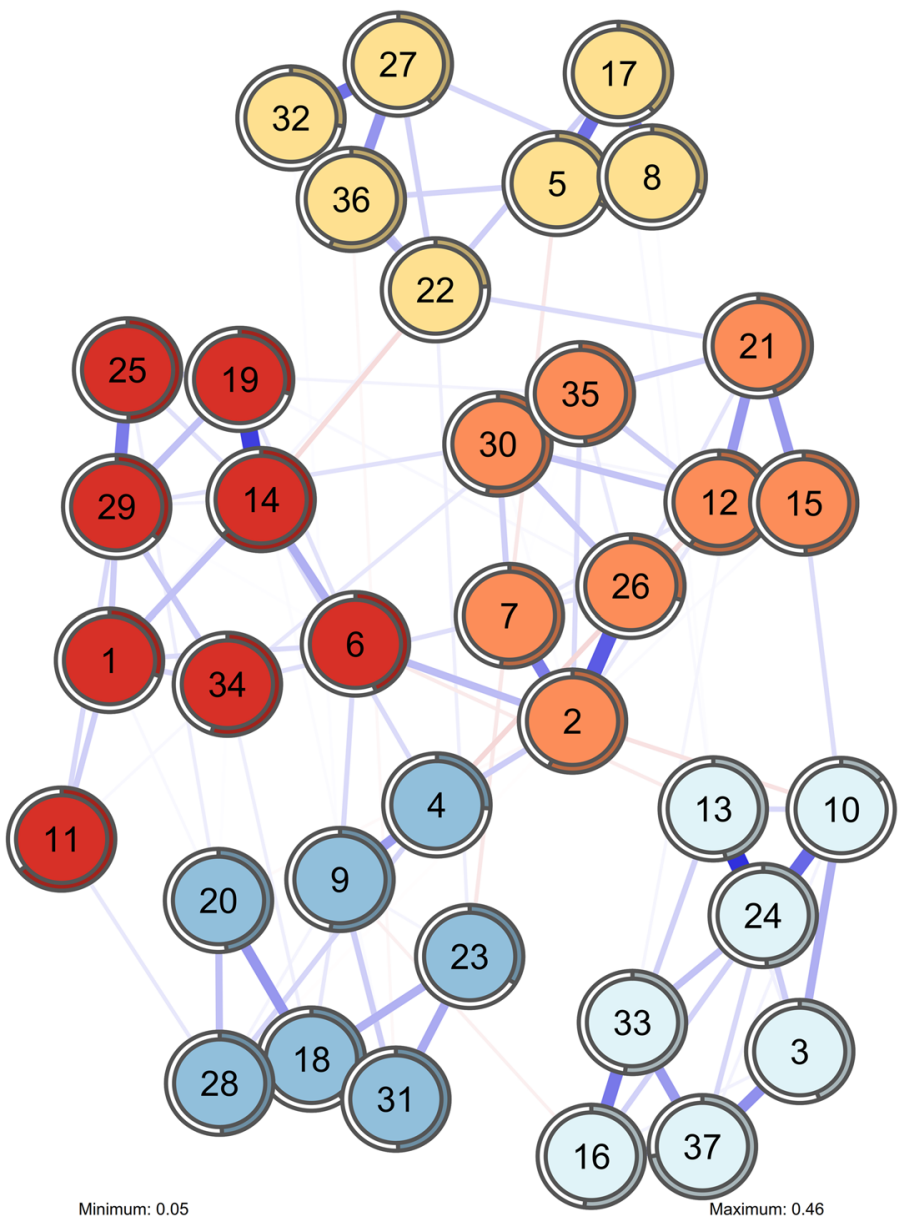

1: Item1

- 6: Item6

11: Item11

- 14: Item15

- 19: Item20

- 25: Item26

- 29: Item31

- 34: Item36

Describe

- 2: Item2

- 7: Item7

- 12: Item12

- 15: Item16

- 21: Item22

- 26: Item27

- 30: Item32

- 35: Item37

Acting with Awareness

o 5: Item5.and.Item13

o 8: Item8

- 17: Item18

- 22: Item23

- 27: Item28

- 32: Item34

○ $36:$ Item38

Non-Judging

- 3: Item3

- 10: Item10

- 13: Item14

- 16: Item17

- 24: Item25.and.Item30

- 33: Item35

- 37: Item39

Non-Reacting

- 4: Item4

- 9: Item 9

- 18: Item19

- 20: Item21

- 23: Item24

- 28: Item29

- 31: Item33 
to traditional latent variable extraction methods, such as Parallel Analysis (Golino et al., 2020). Fourth, we also implemented a community analysis (mathematically equivalent to EGA) comparing other algorithms than the EGA's default (i.e., walktrap and Louvain), like spinglass and multilevel. Model fit was assessed with entropy indices (Golino et al., 2019). More concretely, the Entropy Index (EFI) with Shannon entropy, the EFI with Von Neumann entropy (EFIvn), and the Total EFIvn (TEFIvn). In all cases, lower values indicate a better fit. TEFIvn was prioritized since it shows higher accuracy in over factored solutions. Fifth, we bootstrapped the EGAs to assess its replicability. We implemented two network estimation methods (gLASSO and Triangular Maximally Filtered Graph, or TMFG) crossed with walktrap and Louvain algorithms. Dimension stability was assessed with the median of proportions of proposed solutions with a confidence interval, while item stability was assessed with the median of replicated item correspondences following a certain solution.

Finally, we implemented an Exploratory Factor Analysis for this sample as a comparison to RPCNs and EGA estimates with a more standard technique (see OSF Supplementary Materials for technical details and outputs).

To cross-validate our exploratory results, we implemented confirmatory factor analysis (CFAs) to the second sample with the structures proposed by EGA. As we mentioned before, all our items were Likert-type so we implemented polychoric correlations, except for composite scores of overlapping items. The chosen estimation method was weighted least squares mean-and-variance adjusted (WLSMV) since it provides better performance to other methods, like maximum likelihood robust (MLR) or unweighted least squares (ULS) (Li, 2016). Fit measures were the chi-square statistic (reported but not interpreted), the Comparative Fit Index (CFI) and Tucker-Lewis Index (TLI), both with values $>0.90$ as a good fit, the root mean square error approximation (RMSEA), with values $<0.07$ as a good fit, and the standardized root mean square residual (SRMR), with values $<0.08$ as good fit (following Hair et al., 2014, p. 584). If a CFA model did not meet the fit criteria, we implemented modification indices that provided a theoretical and empirical improvement of the model. A maximum of three modification indices were allowed. We compared CFA models with formal tests as $\Delta \chi^{2}$ (optimized for robust measures), and direct differences between CFI, TLI, RMSEA, and SRMR. As a general assessment, we interpret increases in CFI and TLI and decreases of RMSEA and SRMR as indicative of better fit.

All analyses were computed using the $\mathrm{R}$ environment $(\mathrm{R}$ Development Core Team, 2020). Descriptive statistics were computed using psych (Rewelle, 2014), while the network analyses were computed using bootnet (Epskamp et al., 2018), mgm (Haslbeck \& Waldorp, 2015), and networktools
(Jones, 2020). The EGAs were computed using EGAnet (Golino \& Christensen, 2019), and the CFAs were computed using lavaan (Rosseel, 2012). Data is available at https://osf. io/65abr/ along with scripts and supplementary materials.

\section{Results}

For both samples, item descriptive statistics did not show relevant skewness except for item 23 in sample 2, although they were prone to platykurtic distributions (Table 1). Therefore, we selected the mean and standard deviation (SD) as their central tendency and dispersion estimators. All items had means around 3 (the central category) and SDs around 1. No items showed informativeness issues (none had SDs relevantly below the rest).

Overlapping item analysis proposed two pairs of items as empirically overlapping: Items 5 and 13 ("When I do something, my mind wanders and I get easily distracted," and "I get easily distracted," respectively), and items 25 and 30 ("I tell myself I shouldn't be thinking the way I' $m$ thinking" and "I think some of my emotions are bad or inappropriate and I shouldn't feel them," respectively). Therefore, we merged both pairs into two composite scores. Regarding network analyses, the FFMQ items showed a positively correlated network (Fig. 1) with moderate-to-high predictability (mean of $R^{2}=0.444, \mathrm{SD}=0.126$ ), with only one item showing an $R^{2}$ lower than 0.25 (item $11, R^{2}=0.142$ ). Bootstrap analysis revealed a generally stable network (see OSF Supplementary Materials), with a correlation stability coefficient of 0.75 , which is interpreted as stable. Based on visual inspection, theoretical facets show a good preliminary correspondence within the network, with all items belonging to a certain facet being close to each other and little or no edges connecting between groups.

Multi-dimensional scaling plots (Fig. 2) show a similar behavior, with items belonging to each theoretical facet being close and connected with each other. However, two cautionary patterns arise: First, the Observe and NonReacting facets seem more disperse than the rest of the facets, with some interconnections between them. This could be due to these facets being merged in this sample, although they struggle to find a theoretical meaning following their content. Second, the Acting with Awareness items appears to split into two subgroups, with low correlations between each other. While proximate between them, the first half of items of the Actaware are highly connected between them, while only one weak connection for each item with other Actaware items. The items of each subgroup are the first half of the Actaware facet (5 and 13 composite, 8, and 18) and the second half (23, 28,34 , and 38). Attending to the content of the items, a plausible interpretation can be made. While both measure 
Table 1 Descriptive statistics of the FFMQ

\begin{tabular}{|c|c|c|c|c|c|c|c|c|}
\hline \multirow[b]{2}{*}{ Items } & \multicolumn{4}{|c|}{ Sample 1} & \multicolumn{4}{|c|}{ Sample 2} \\
\hline & Mean & $\mathrm{SD}$ & $\begin{array}{l}\text { Skewness } \\
(\mathrm{SE}=0.07)\end{array}$ & $\begin{array}{l}\text { Kurtosis } \\
(\mathrm{SE}=0.15)\end{array}$ & Mean & SD & $\begin{array}{l}\text { Skewness } \\
(\mathrm{SE}=0.07)\end{array}$ & $\begin{array}{l}\text { Kurtosis } \\
(\mathrm{SE}=0.14)\end{array}$ \\
\hline Average & 3.16 & 1.08 & 0.14 & -0.56 & 3.38 & 1.13 & -0.34 & -0.51 \\
\hline 1 & 2.72 & 1.06 & -0.30 & -0.70 & 3.18 & 1.15 & -0.06 & -0.80 \\
\hline 2 & 3.25 & 1.13 & 0.11 & -0.97 & 3.20 & 1.17 & -0.14 & -0.82 \\
\hline 3 & 2.90 & 1.19 & -0.01 & -0.46 & 3.06 & 1.27 & -0.06 & -1.04 \\
\hline 4 & 2.81 & 0.98 & 0.16 & -0.74 & 3.19 & 1.01 & -0.08 & -0.29 \\
\hline 5 & 2.67 & 1.10 & 0.03 & -0.95 & 3.26 & 1.14 & -0.39 & -0.61 \\
\hline 6 & 2.91 & 1.17 & -0.45 & -0.41 & 3.42 & 1.24 & -0.28 & -1.00 \\
\hline 7 & 3.53 & 1.04 & -0.27 & -0.57 & 3.40 & 1.13 & -0.29 & -0.70 \\
\hline 8 & 3.15 & 1.05 & 0.15 & -0.38 & 3.68 & 1.12 & -0.68 & -0.25 \\
\hline 9 & 2.82 & 0.96 & 0.01 & -0.90 & 3.40 & 1.06 & -0.30 & -0.51 \\
\hline 10 & 3.11 & 1.19 & 0.02 & -1.04 & 3.26 & 1.23 & -0.27 & -0.89 \\
\hline 11 & 2.94 & 1.24 & -0.59 & -0.28 & 2.81 & 1.35 & 0.19 & -1.17 \\
\hline 12 & 3.58 & 1.06 & 0.10 & -0.78 & 3.46 & 1.17 & -0.41 & -0.65 \\
\hline 13 & 2.75 & 1.09 & -0.35 & -0.84 & 3.43 & 1.16 & -0.52 & -0.51 \\
\hline 14 & 3.46 & 1.19 & -0.31 & -0.76 & 3.76 & 1.18 & -0.79 & -0.20 \\
\hline 15 & 3.19 & 1.16 & -0.54 & -0.35 & 3.51 & 1.24 & -0.37 & -0.95 \\
\hline 16 & 3.55 & 1.08 & 0.13 & -0.79 & 3.55 & 1.11 & -0.46 & -0.50 \\
\hline 17 & 2.93 & 1.13 & -0.20 & -0.55 & 2.55 & 1.15 & 0.41 & -0.62 \\
\hline 18 & 3.19 & 1.03 & -0.10 & -0.63 & 3.85 & 1.02 & -0.81 & 0.18 \\
\hline 19 & 3.00 & 1.04 & -0.14 & -0.74 & 3.33 & 1.11 & -0.35 & -0.59 \\
\hline 20 & 3.19 & 1.10 & -0.10 & -0.56 & 3.46 & 1.20 & -0.31 & -0.88 \\
\hline 21 & 3.13 & 1.02 & -0.62 & 0.02 & 3.19 & 1.10 & -0.15 & -0.61 \\
\hline 22 & 3.54 & 0.98 & -0.17 & -0.73 & 3.75 & 1.01 & -0.66 & -0.03 \\
\hline 23 & 3.34 & 1.11 & 0.32 & -0.65 & $4.00^{\mathrm{a}}$ & 0.95 & -1.27 & 1.27 \\
\hline 24 & 2.61 & 1.10 & -0.32 & -0.72 & 3.03 & 1.13 & -0.19 & -0.70 \\
\hline 25 & 3.38 & 1.15 & -0.46 & -0.50 & 3.43 & 1.13 & -0.38 & -0.61 \\
\hline 26 & 3.65 & 1.02 & -0.25 & -0.72 & 3.94 & 1.05 & -0.78 & -0.14 \\
\hline 27 & 3.31 & 1.08 & -0.11 & -0.48 & 3.22 & 1.13 & -0.20 & -0.72 \\
\hline 28 & 3.05 & 0.99 & 0.15 & -0.39 & 3.85 & 0.96 & -0.77 & 0.38 \\
\hline 29 & 2.77 & 0.93 & -0.32 & -0.82 & 2.96 & 1.12 & 0.07 & -0.69 \\
\hline 30 & 3.42 & 1.17 & -0.45 & -0.62 & 3.64 & 1.17 & -0.62 & -0.45 \\
\hline 31 & 3.51 & 1.13 & -0.34 & -0.60 & 3.61 & 1.21 & -0.48 & -0.79 \\
\hline 32 & 3.49 & 1.05 & 0.090 & -0.50 & 3.01 & 1.10 & 0.05 & -0.71 \\
\hline 33 & 2.80 & 0.98 & -0.10 & -0.55 & 2.33 & 1.17 & 0.48 & -0.70 \\
\hline 34 & 3.02 & 1.02 & -0.11 & -0.82 & 4.06 & 0.93 & -0.89 & 0.40 \\
\hline 35 & 3.28 & 1.12 & -0.52 & -0.25 & 3.21 & 1.20 & -0.11 & -0.91 \\
\hline 36 & 3.61 & 0.98 & -0.20 & -0.63 & 3.58 & 1.11 & -0.53 & -0.42 \\
\hline 37 & 3.33 & 1.06 & 0.00 & -0.65 & 3.05 & 1.13 & 0.06 & -0.80 \\
\hline 38 & 2.95 & 1.05 & -0.13 & -0.92 & 3.90 & 1.02 & -0.80 & 0.12 \\
\hline 39 & 3.23 & 1.18 & 0.14 & -0.56 & 3.24 & 1.22 & -0.22 & -0.87 \\
\hline
\end{tabular}

Note. SD, statistical deviation; $S E$, standard error

${ }^{a}$ Median since skewness is $>|1|$ components of mindlessness or "automatic pilot," the first subgroup seem to be more related to distractibility (e.g., item 13: "I am easily distracted"), and the second subgroup to mindless actions (e.g., item 38: "I find myself doing things without paying attention"). Item distribution with this proposed split is displayed in Table 2. Finally, connections between theoretical facets are few and low, except for item 2.

Attending the rest of the network, the Describe and NonJudging items could display a behavior like Actaware but 
Fig. 2 Estimated network of the FFMQ with the 5-facet solution-multi-dimensional scaling method. Note: Items are rounded in circles, with pies representing the explained variance $\left(R^{2}\right)$ of each item. Item size is reduced to make easier interpretations. Lines connecting items represent correlations, blue $=$ positive correlation, red = negative correlation. Highly correlated items tend to be closer, while non-correlated nodes tend to be farther

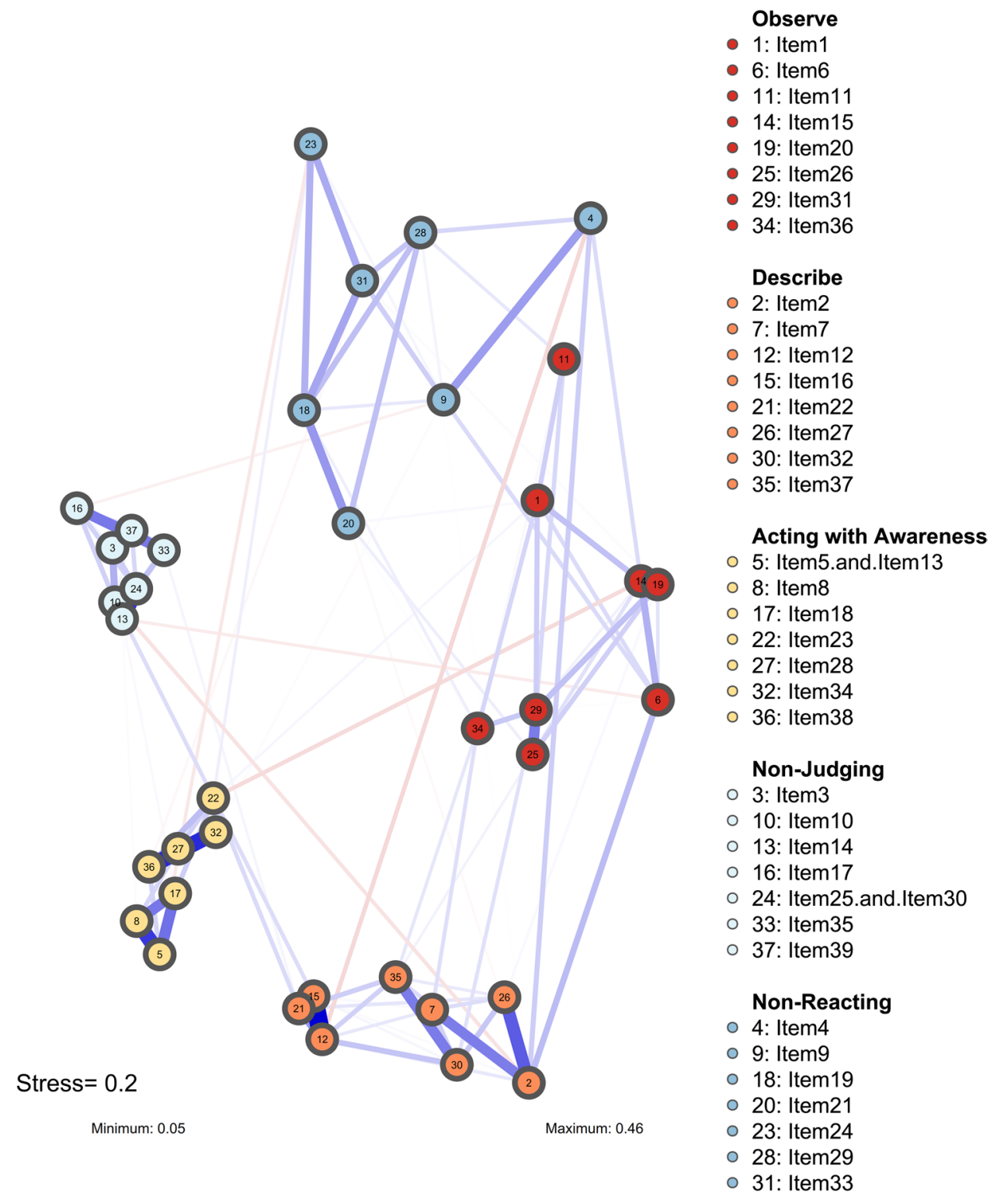

Table 2 Contents of the Acting with Awareness items with a proposed interpretation

\begin{tabular}{lll}
\hline Acting with Awareness items & Contents & Proposed interpretation \\
\hline $\begin{array}{l}\text { 5. When I do things, my mind wanders off and I'm easily distracted } \\
\begin{array}{l}\text { 8. I don't pay attention to what I'm doing because I'm daydreaming, worrying, } \\
\text { or otherwise distracted }\end{array}\end{array}$ & $\begin{array}{l}\text { Distraction, actions, mind-wandering } \\
\text { Distraction, inattentiveness, actions, } \\
\text { mind-wandering } \\
\text { 13. I am easily distracted }\end{array}$ \\
$\begin{array}{l}\text { 18. I find it difficult to stay focused on what's happening in the present } \\
\text { Inattentiveness, present moment }\end{array}$ \\
$\begin{array}{l}\text { 23. It seems I am "running on automatic" without much awareness of what I'm } \\
\text { doing }\end{array}$ & Actions, automatic pilot \\
$\begin{array}{l}\text { 28. I rush through activities without being really attentive to them } \\
\text { 34. I do jobs or tasks automatically without being aware of what I'm doing }\end{array}$ & $\begin{array}{l}\text { Actions, inattentiveness } \\
\text { Actions, automatic pilot }\end{array}$ \\
38. I find myself doing things without paying attention & Actions, inattentiveness
\end{tabular}


were not regarded in this way due to more connections between items. Therefore, we conclude that the theoretical five facets seem to hold, but two alternative models arise as potentially meaningful: A 4-facet model with Observe and Non-Reacting merged into a single facet (Fig. 3), and a 6-facet model with Actaware split in two (Fig. 4).

\section{Exploratory Graph Analysis}

The default EGA supported the 5-facet model for the FFMQ. Different number of steps in the walktrap algorithm threw the same solution except for 8 and 9 steps, proposing the 4-facet solution. Regarding the spinglass algorithm, a different outlook was displayed. While the default algorithm proposed the 5-facet model, most variations of spins (from 25 to 500 spins by steps of 50 stems) proposed the 6-facet model. Finally, the multilevel algorithm proposed the 5 -facet model without variations.

Entropy indices were convergent with the walktrap proposal, getting the best fit for the 5 -facet model (Table 3 ). The bootstrap analysis proposed in all cases the 5 -facet solution with confidence intervals not reaching values below 4 or above 6 (see OSF Supplementary Materials). The 4 and 6-facet models received very little support. Dimension and item stability analysis reported high stability for almost all facets in all cases. The only exception was Non-Reacting, due to low or medium recovery rates for item 4 , with the rest of the items displaying acceptable or good recovery rates. Therefore, EGA proposes the 5-facet structure as the most fitting and stable solution for the FFMQ.

Finally, regarding the Exploratory Factor Analysis, no clear support was provided for the 4,5 or 6 -facet model (see OSF Supplementary Materials). Nevertheless, the 5-facet model is perhaps the most fitting model due to partial support of Parallel Analysis and factor loadings following a simple structure with theoretical meaning.

\section{Confirmatory Factor Analysis}

As mentioned before, we imported the three resulting solutions from EGA and applied CFAs to cross-validate its results in the second sample. Fit measures are displayed in
Fig. 3 Networks of the FFMQ with 4-facet solution. Note: Items are rounded in circles, with pies representing the explained variance $\left(R^{2}\right)$ of each item. Lines connecting items represent correlations, blue $=$ positive correlation, red = negative correlation. Highly correlated items tend to be closer, while non-correlated nodes tend to be farther

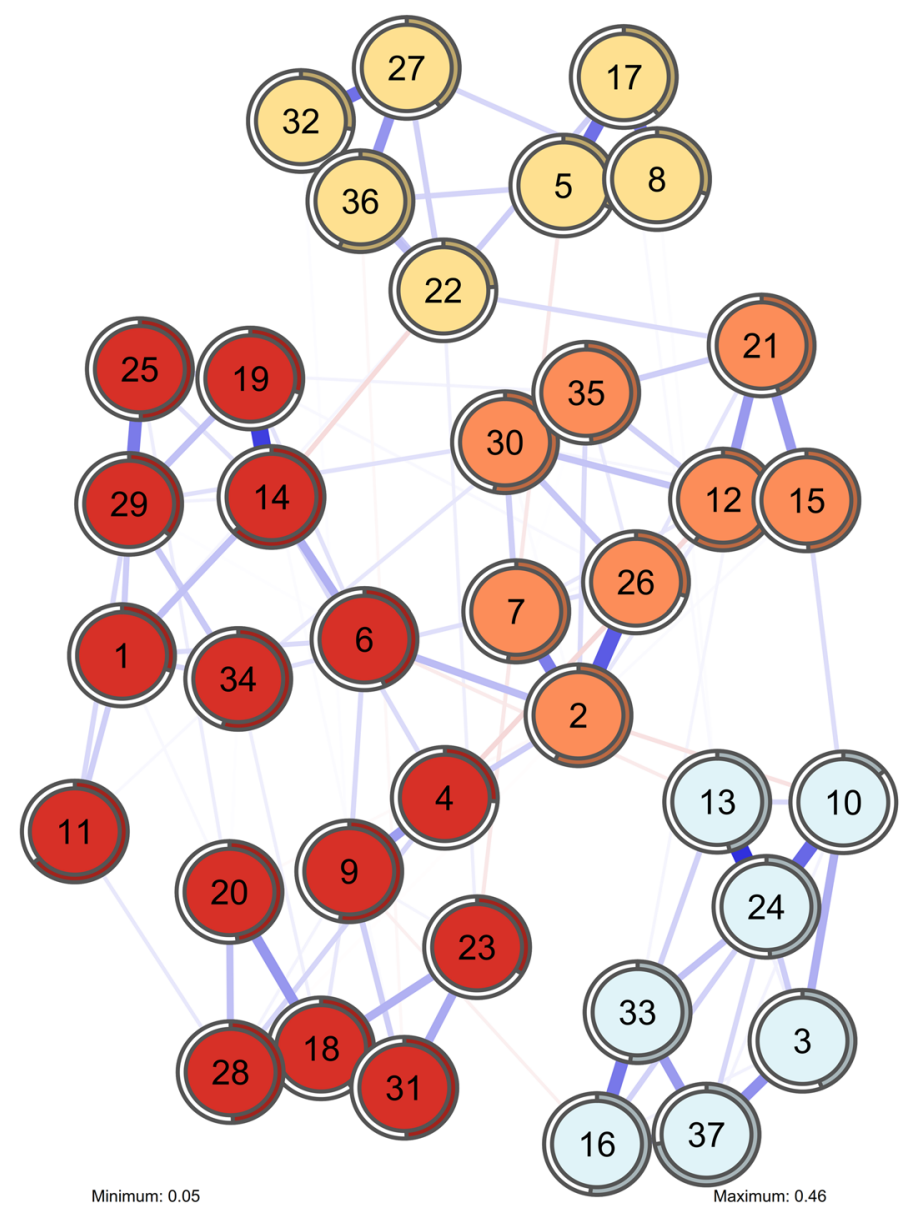

Observe and Non-Reacting

- 1: Item1

- 6: Item6

11: Item11

- 14: Item15

- 19: Item20

- 25: Item26

- 29: Item31

- 34: Item36

- 4 : Item4

- 9: Item9

- 18: Item19

- 20: Item21

- 23: Item24

- 28: Item29

- 31: Item33

Describe

- 2: Item2

- 7: Item7

- 12: Item12

- 15: Item16

- 21: Item22

- 26: Item27

- 30: Item32

- 35: Item37

Acting with Awareness

- 5: Item5.and.Item13

- 8: Item8

- 17: Item18

- 22: Item23

- 27: Item28

- 32: Item34

○ 36: Item38

\section{Non-Judging}

- 3: Item3

- 10: Item10

- 13: Item14

- 16: Item17

- 24: Item25.and.Item30

- 33: Item35

- 37: Item39 
Fig. 4 Networks of the FFMQ with 6-facet facet solution. Note: Items are rounded in circles, with pies representing the explained variance $\left(R^{2}\right)$ of each item. Lines connecting items represent correlations, blue $=$ positive correlation, red $=$ negative correlation. Highly correlated items tend to be closer, while non-correlated nodes tend to be farther

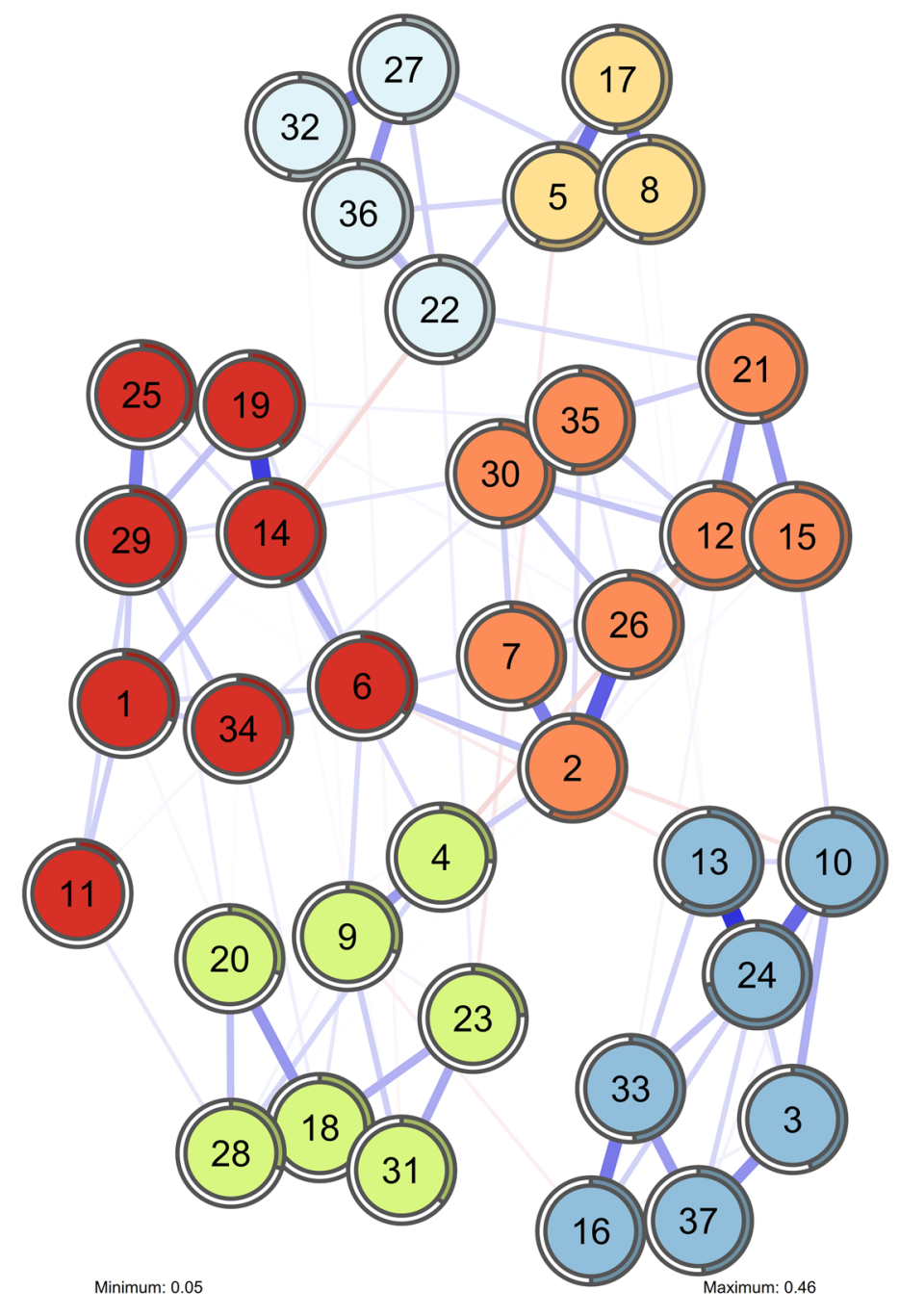

Observe

- 1: Item1

- 6: Item6

- 11: Item11

- 14: Item15

- 19: Item20

- 25: Item26

- 29: Item31

- 34: Item36

Describe

- 2: Item2

- 7: Item7

- 12: Item12

- 15: Item16

- 21: Item22

- 26: Item27

- 30: Item32

- 35: Item37

Distractability

- 5: Item5.and.Item13

- 8: Item8

○ 17: Item18

\section{Mindless Actions}

- 22: Item23

- 27: Item28

- 32: Item34

- 36: Item38

\section{Non-Judging}

- 3: Item3

- 10: Item10

- 13: Item14

- 16: Item17

- 24: Item25.and.Item30

- 33: Item35

- 37: Item39

\section{Non-Reacting}

- 4: Item4

- 9: Item9

- 18: Item19

- 20: Item21

- 23: Item24

- 28: Item29

- 31: Item33
Table 3 Entropy fit indices for three different facet solutions of the FFMQ

\begin{tabular}{llll}
\hline Number of facets & EFI & EFIvn & TEFIvn \\
\hline 4 facets (O and NR fused) & -0.81 & 3.43 & -47.13 \\
5 facets & $-1.07^{*}$ & 3.39 & $-52.75^{*}$ \\
6 facets (AW split in two) & -1.05 & $2.80^{*}$ & -51.69 \\
\hline
\end{tabular}

Note. Lower values indicate a better fit. The 5-facet solution is proposed by literature and EGA with walktrap and multilevel algorithms. The 6-facet solution is proposed by EGA with spinglass algorithm and derived from descriptive results

$O$, observe; $N R$, non-reacting to inner experience; $A W$, Acting with Awareness

*Best fitting model
Table 4. None of the raw models displayed a good fit. Thus, we examined modification indices. We found three crossloadings that improved fit: (1) Item 17 measuring Observe, which can be explained as that being attentive would benefit directly the tendency to label thoughts as good or bad since it measures internal monitoring; (2) Item 22 measuring Non-Judging, which can be explained as that a non-judging attitude would benefit directly the descriptions of sensations since it's a more neutral approach to experience; and (3) Item 16 measuring Non-Judging, which can be explained similarly than item 22, but with emotions instead of sensations. By adding modification indices, model fit was appropriate for CFI, but not for TLI, RMSEA, and SRMR in all models. However, fit comparisons offered a significant improvement for all models (all $\Delta \chi^{2}$ with $p<0.001$ ), and with improvements in all fit indices ( $\Delta$ CFI, $\Delta$ TLI, $\triangle$ RMSEA, and $\Delta$ SRMR). Therefore, both the 5-facet and 6-facet structures 


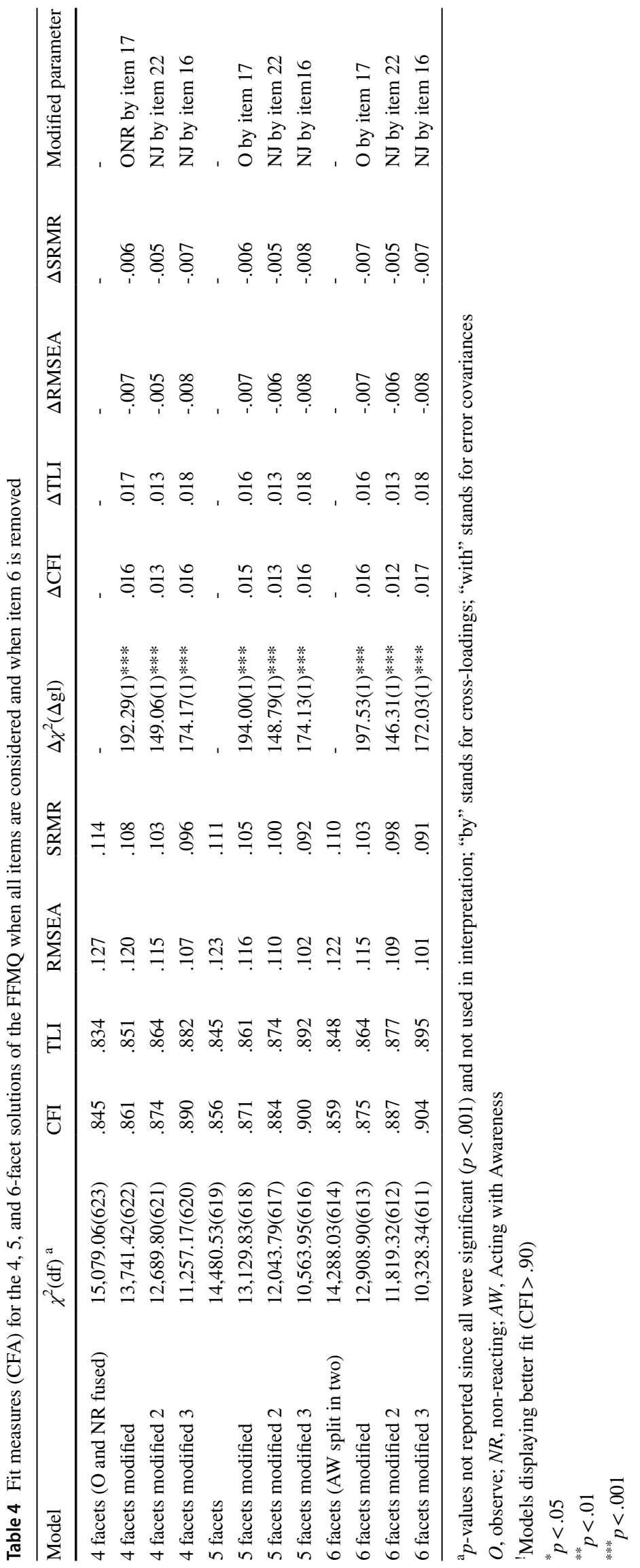


provided a questionable fit. Factor loadings were positive, significant, and above 0.3 in all models, and all covariances between factors were positive and significant. This suggests a potential second-order latent solution, thus we estimated a hierarchical model. However, fit measures were not satisfactory $\left(\chi^{2}(624)=13,028.06, p<0.001, \mathrm{CFI}=0.687\right.$, $\mathrm{TLI}=0.666$, RMSEA $=0.118, \mathrm{SRMR}=0.126$ ), even when modification indices were considered. Therefore, we discard a second-order stable structure.

To conclude this section, cross-validation results indicated a questionable fit of FFMQ in all models, although support partially the 5 and 6-facet models with minor modifications.

\section{Discussion}

This study aimed to explore the FFMQ facets to generate an interpretable solution for existing and future research. To do so, we implemented RPCNs and EGAs to explore and contrast optimal solutions for a large sample. Results show that the 5-facet solution produces a suitable network supported by EGA, although two alternative structures are also proposed: A 4-facet structure, merging Observe and Non-Reacting, and a 6-facet structure, splitting Acting with Awareness in two, named Distractibility and Mindless Actions.

As regards the 4-facet solution, the Observe and NonReacting facets were merged. Technically, results suggest items of both facets could be considered measuring the same phenomenon. One possible interpretation is a theoretically authentic latent variable, although it is difficult to label and interpret. We could think that these items assess a contemplative mindset characterized by an equanimity attitude to the phenomena that constitute the experience (i.e., sensations, thoughts, emotions, sounds). As a whole, these items seem to evaluate the tendency to notice and pay attention to internal and external phenomena, establishing a relationship with them characterized by decentering (i.e., the meta-cognitive observation of the contents of consciousness; Bernstein et al., 2015), and detachment (i.e., not-identification with the contents of the consciousness). Both processes would reduce the tendency to react to thoughts, feelings, and emotions and promotes the tendency to open to them, and allow and let them be without a reactive attitude. In other words, these items of the FFMQ seem to evaluate the ability of the individual to remain present in an attitude of equanimity. We understand equanimity as "an even-minded mental state or dispositional tendency toward all experiences or objects, regardless of their affective valence (pleasant, unpleasant or neutral) or source ... (that) allows awareness to be even and unbiased by facilitating an attitude of non-attachment and nonresistance" (Desbordes et al., 2015, p. 357-358).
Another possibility is that those items represent a method factor. The Observe facet is known for its mixed functioning. Also, the Non-Reacting facet could present a mixed behavior due to a lack of comprehension of items in a general population sample, as suggested in previous studies (Lecuona et al., 2020) and stability analysis of EGA. It could be the most distant facet to the Western culture to understand, therefore prone to be influenced by confusion or response biases. Generically, the western population experiences that thoughts and emotions constitute the self, and they do not experience the feelings, emotions, and thoughts as phenomena that flow through the field of consciousness that are possible to observe (Roeser \& Peck, 2009). The fact of not experiencing the "self-as-an-observant" of internal phenomena can make it challenging to understand the items of the Non-reacting facet since they refer to relate with thoughts and emotions from a "self-as-an-observant" (e.g., item 9, "I watch my feelings without getting lost in them" or item 19, "when I have distressing thoughts or images, I step back and am aware of the thought or image without getting taken over by it"). Thus, this merged facet of Observe and NonReact could represent a general bias latent factor of mixed or confused item responses. Nevertheless, both hypotheses fail to provide substantive arguments to illustrate the common grounds between these two phenomena. In addition, more research is needed on the cross-cultural potential differences on the FFMQ and mindfulness phenomena (Karl et al., 2020).

Regarding the 6-facet solution, the Actaware facet split into two sub-facets. A content analysis revealed the best labels were Distractibility (i.e., tendency or proneness to distraction) and Mindless actions (this is, tendency or proneness to perform actions without conscience of doing them). One similar phenomenon to mindless actions is the "automatic pilot" concept (Kabat-Zinn, 1990), but this characteristic appeared to be in both sub-facets, as revealed by the content analysis. From a theoretical point of view, we can distinguish distractibility and mindless actions, although it might not be essentially different phenomena, or at least, they would be overlapped. However, this solution has been replicated in a recent cross-cultural comparison (Karl et al., $2020)$ with a large sample size $(N>8000)$. Thus, more research is needed to ensure the likelihood of this structure.

The EGA displays the 4 and 6 -facet solutions as unstable and more disorganized in this sample, prioritizing the 5-facet structure. Nevertheless, the CFA prioritized almost equally the 5-facet and 6-facet solutions, while content analysis converged with CFA. Therefore, we conclude that while the 5 -facet solution is the standard of mindfulness literature, both solutions count with strong evidence supporting them. Thus, the 6-facet solution provides a competitive alternative to the 5-facet solution. We conclude that the data does not fully support neither structure, thus we call for caution and 
encourage future studies to explore the replicability of our findings. In addition, this study points out that the internal validity of the FFMQ is still unclear and more developments (evidence and improved instruments) are required in future studies. In this sense, a promising area of development is behavioral instruments since they are less biased by cultural or verbal representations. Examples of these instruments are the breath-counting task (Isbel et al., 2020; Levinson et al., 2014) or the simulated thoughts paradigm (Amir et al., 2021; for a review, see Hadash \& Bernstein, 2019). However, this research field is in early development. Future studies should expand it and aim to develop theoretically and empirically robust behavioral instruments for mindfulness.

We propose some guidelines to make optimal use of the FFMQ. First, we advise considering complementary mindfulness instruments, like the Freiburg Mindfulness Questionnaire (FMI, Sauer et al., 2013) or the Comprehensive Inventory of Mindfulness Experiences (CHIME- $\beta$, Bergomi et al., 2013a). In this sense, we prioritize psychometric stability over losing theoretical richness, but we leave that to the discretion of the user. Second, we recommend scoring facets of the FFMQ, but not the overall mindfulness facet. The reason to advice against the overall mindfulness factor is the lack of fit when including it that is supported by other studies as well (e.g. Karl et al., 2020; Lecuona et al., 2020). Finally, we advise researchers to test the 5 and 6-facet models when analyzing samples with the FFMQ and choosing the model with best fit, since literature seems to support both structures.

To conclude, this study shows partial support for the 5-facet model of the FFMQ. However, an alternative model of 6 facets (with the Acting with Awareness split in two) shows similar support. Mindfulness researchers and applied users should take caution in using the FFMQ and consider using other self-reports (e.g., FMI or CHIME- $\beta$ ), and if using it, assess the most fitting option between scoring the five or six facets, instead of the overall mindfulness factor.

\section{Limitations and Future Research}

This study has some limitations. First, the design was crosssectional, which obscures possible longitudinal features of the FFMQ, i.e., invariance between pre and post participation in a mindfulness-based intervention. Also, all our measures were self-reports, which impedes control for single method bias. Another limitation is that the FFMQ was the only psychological instrument included in the study, which hinders the possibility of exploring relations of the FFMQ with other relevant constructs. Finally, although both sample sizes were large, future studies can assess meditation practice as a possible moderator of the latent facets and their interactions. Future research should also focus on finding more robust ways of assessing mindfulness, and if the FFMQ is used, explore the most suitable model for that specific data.

Author Contribution OL designed and executed the study, analyzed the data, wrote the paper and responses to peer-review, and supervised the peer-review contributions. CGR assisted the interpretation and discussion of results, provided critical feedback and helped shape the manuscript, and assisted the responses to peer-review. SdR provided critical feedback and helped shape the manuscript, and assisted the responses to peer-review. JEMJ provided critical feedback and helped shape the manuscript, and assisted the responses to peer-review. RMML provided the data of the second sample. RRC assisted the design and execution of the study provided critical feedback and helped shape the manuscript, and assisted the responses to peer-review.

Funding Open Access funding provided thanks to the CRUE-CSIC agreement with Springer Nature.

\section{Declarations}

Ethics Approval All procedures performed in this study were following the ethical standards of the two institutional research committees (King Juan Carlos University; Autonomous University of Madrid) and with the Helsinki declaration and its later amendments or comparable ethical standards.

Informed Consent Informed consent was obtained from all individual participants included in the studies.

Conflict of Interest The authors declare no competing interests.

Open Access This article is licensed under a Creative Commons Attribution 4.0 International License, which permits use, sharing, adaptation, distribution and reproduction in any medium or format, as long as you give appropriate credit to the original author(s) and the source, provide a link to the Creative Commons licence, and indicate if changes were made. The images or other third party material in this article are included in the article's Creative Commons licence, unless indicated otherwise in a credit line to the material. If material is not included in the article's Creative Commons licence and your intended use is not permitted by statutory regulation or exceeds the permitted use, you will need to obtain permission directly from the copyright holder. To view a copy of this licence, visit http://creativecommons.org/licenses/by/4.0/.

\section{References}

American Mindfulness Research Association (AMRA) (2021). Mindfulness publications by year, 1980-2020. Retrieved March 10, 2019, from https://goamra.org/Library.

Amir, I., Ruimi, L., \& Bernstein, A. (2021). Simulating thoughts to measure and study internal attention in mental health. Scientific Reports, 11(1), 1-17. https://doi.org/10.1038/ s41598-021-81756-w

Baer, R. A., Smith, G. T., Lykins, E., Button, D., Krietemeyer, J., Sauer, S., Walsh, E., Duggan, D., \& Williams, J. M. G. (2008). Construct validity of the five facet mindfulness questionnaire in meditating and nonmeditating samples. Assessment, 15(3), 329-342. https:// doi.org/10.1177/1073191107313003

Belzer, F., Schmidt, S., Lucius-Hoene, G., Schneider, J. F., OrellanaRios, C. L., \& Sauer, S. (2013). Challenging the construct validity 
of mindfulness assessment-A cognitive interview study of the freiburg mindfulness inventory. Mindfulness, 4(1), 33-44. https:// doi.org/10.1007/s12671-012-0165-7

Bergomi, C., Tschacher, W., \& Kupper, Z. (2013a). Measuring mindfulness: First steps towards the development of a comprehensive mindfulness scale. Mindfulness, 4(1), 18-32. https://doi.org/10. 1007/s12671-012-0102-9

Bergomi, C., Tschacher, W., \& Kupper, Z. (2013b). The assessment of mindfulness with self-report measures: Existing scales and open issues. Mindfulness, 4(3), 191-202. https://doi.org/10.1007/ s12671-012-0110-9

Bernstein, A., Hadash, Y., Lichtash, Y., Tanay, G., Shepherd, K., \& Fresco, D. M. (2015). Decentering and related constructs. Perspectives on Psychological Science, 10(5), 599-617. https://doi. org/10.1177/1745691615594577

Bringmann, L., Elmer, T., Epskamp, S., Krause, R., Schoch, D., Wichers, M., Wingman, J. T. W., \& Snippe, E. (2019). What do centrality measures measure in psychological networks? Journal of Abnormal Psychology, 128(8), 892-903. https://doi.org/10.1037/ abn0000446

Carpenter, J. K., Conroy, K., Gomez, A. F., Curren, L. C., \& Hofmann, S. G. (2019). The relationship between trait mindfulness and affective symptoms: A meta-analysis of the five facet mindfulness questionnaire (FFMQ). Clinical Psychology Review, 74, 101785. https://doi.org/10.1016/j.cpr.2019.101785

Cebolla, A., García-Palacios, A., Soler, J., Guillen, V., Baños, R., \& Botella, C. (2012). Psychometric properties of the spanish validation of the five facets mindfulness questionnaire (FFMQ). The European Journal of Psychiatry, 26(2), 118-126. https://doi.org/ 10.4321/S0213-61632012000200005

Creswell, J. D. (2016). Mindfulness interventions. Annual Review of Psychology, 68, 491-516. https://doi.org/10.1146/annur ev-psych-042716-051139

Desbordes, G., Gard, T., Hoge, E. A., Hölzel, B. K., Kerr, C., Lazar, S. W., Olendzki, A., \& Vago, D. R. (2015). Moving beyond mindfulness: Defining equanimity as an outcome measure in meditation and contemplative research. Mindfulness, 6(2), 356-372. https:// doi.org/10.1007/s12671-013-0269-8

Epskamp, S., \& Fried, E. I. (2018). A tutorial on regularized partial correlation networks. Psychological Methods, 23(4), 617-634. https://doi.org/10.1037/met0000167

Epskamp, S., Borsboom, D., \& Fried, E. I. (2018). Estimating psychological networks and their accuracy: A tutorial paper. Behavior Research Methods, 50(1), 195-212. https://doi.org/10.3758/ s13428-017-0862-1

Goldberg, S. B., Wielgosz, J., Dahl, C., Schuyler, B., MacCoon, D. S., Rosenkranz, M., Lutz, A., Sebranek, C. A., \& Davidson, R. J. (2015). Does the five facet mindfulness questionnaire measure what we think it does? Construct validity evidence from an active controlled randomized clinical trial. Psychological Assessment, 28(8), 1009-1014. https://doi.org/10.1037/pas0000233

Golino, H., Shi, D., Garrido, L., Christensen, A., Nieto, M., Sadana, R., Thiyagarajan, J. A., \& Pérez-Molina, A. (2020). Investigating the performance of exploratory graph analysis and traditional techniques to identify the number of latent factors: A simulation and tutorial. Psychological Methods, 25(3), 292-320. https://doi. org $/ 10.1037 /$ met0000255

Golino, H., \& Christensen, A. (2019). EGAnet: Exploratory graph analysis-a framework for estimating the number of dimensions in multivariate data using network psychometrics. $\mathrm{R}$ package version 0.8.0.

Golino, H. F., \& Epskamp, S. (2017). Exploratory graph analysis: A new approach for estimating the number of dimensions in psychological research. PLoS ONE, 12(6), e0174035. https://doi.org/10. 1371/journal.pone.0174035
Golino, H., Moulder, R., Shi, D., Christensen, A. P., Nieto, M. D., Nesselroade, J. R., Sadana, R., Thiyagarajan, J. A., \& Boker, S. M. (2019). Entropy fit index: New fit measures for assessing the structure and dimensionality of multiple latent variables. Multivariate Behavioral Research. https://doi.org/10.1080/00273171. 2020.1779642

Hadash, Y., \& Bernstein, A. (2019). Behavioral assessment of mindfulness: Defining features, organizing framework, and review of emerging methods. Current Opinion in Psychology, 28, 229-237. https://doi.org/10.1016/j.copsyc.2019.01.008

Hair, J. F., Black, W. C., Babin, B. J., \& Anderson, R. E. (2014). Multivariate data analysis. Pearson Education Limited.

Haslbeck, J. M. B., \& Waldorp, L. J. (2015). mgm: Estimating timevarying mixed graphical models in high-dimensional data. Journal of Statistical Software. http://arxiv.org/abs/1510.06871.

Isbel, B., Stefanidis, K., \& Summers, M. J. (2020). Assessing mindfulness: Experimental support for the discriminant validity of breath counting as a measure of mindfulness but not self-report questionnaires. Psychological Assessment, 32(12), 1184. https://doi.org/ 10.1037/pas0000957

Jones, P. (2020). networktools: Tools for identifying important nodes in networks. R package, version 1.2.3. https://cran-r-project.org/ web/packages/networktools/.

Kabat-Zinn, J. (1990). Full catastrophe living: Using the wisdom of your body and mind to face stress, pain, and illness. Bantam Dell.

Karl, J. A., Méndez Prado, S. M., Gračanin, A., Verhaegen, P., Ramos, A., Prasun Mandal, S., Michalak, J., Zhang, C.-Q., Schmidt, C., Tran, U. S., Druica, E., Solem, S., Astani, A., Xinghua, L., Luciano, J. V., Tkalčić, M., Lilja, J. L., Dundas, I., Wong, S. Y. S., \& Fischer, R. (2020). The cross-cultural validity of the fivefacet mindfulness questionnaire across 16 countries. Mindfulness, 11(5), 1226-1237. https://doi.org/10.1007/s12671-020-01333-6

Lecuona, O., García-Garzón, E., García-Rubio, C., \& RodríguezCarvajal, R. (2020). A psychometric review and conceptual replication study of the five facets mindfulness questionnaire latent structure. Assessment, 27(5), 859-872. https://doi.org/10.1177/ 1073191119873718

Levinson, D. B., Stoll, E. L., Kindy, S. D., Merry, H. L., \& Davidson, R. J. (2014). A mind you can count on: Validating breath counting as a behavioral measure of mindfulness. Frontiers in Psychology, 5, 1202. https://doi.org/10.3389/fpsyg.2014.01202

Li, C.-H. (2016). Confirmatory factor analysis with ordinal data: Comparing robust maximum likelihood and diagonally weighted least squares. Behavior Research Methods, 48(3), 936-949. https://doi. org/10.3758/s13428-015-0619-7

Mcneish, D. (2017). Thanks coefficient alpha, we'll take it from here. Psychological Methods., 23(3), 412-433. https://doi.org/10.1037/ met0000144

Meda, R. M., Herrero, M., Blanco-Donoso, L. M., Moreno-Jiménez, B., \& Palomera, A. (2015). Propiedades psicométricas del "cuestionario de cinco facetas de la conciencia plena" (five facets mindfulness questionnaire, FFMQ-M) en México. Behavioral Psychology, 23(3), 467-487.

Mullarkey, M. C., Stewart, R. A., Wells, T. T., Shumake, J., \& Beevers, C. G. (2018) Self-dislike and sadness are central symptoms of depression in college students: A network analysis. PsyArXiv, osf. io/fujmb.

R Development Core Team (2020). R: A language and environment for statistical computing. Vienna, Austria: R Foundation for Statistical Computing. Retrieved from http://www.r-project.org/.

Rewelle, W. (2014). psych. In Procedures for personality and psychological research. Northwestern University, Evanston, Illinois, USA: Version 1.4.8. Retrieved from http://cran.r-project.org/packa ge $=$ psych.

Roeser, R. W., \& Peck, S. C. (2009). An education in awareness: Self, motivation, and self-regulated learning in contemplative 
perspective. Educational Psychologist, 44(2), 119-136. https:// doi.org/10.1080/00461520902832376

Rosseel, Y. (2012). lavaan: An R package for structural equation modeling. Journal of Statistical Software, 48(2), 1-36. Retrieved from http://www.jstatsoft.org/v48/i02/.

Sauer, S., Ziegler, M., Danay, E., Ives, J., \& Kohls, N. (2013). Specific objectivity of mindfulness: A Rasch analysis of the freiburg mindfulness inventory. Mindfulness, 4, 45-54. https://doi.org/10. 1007/s12671-012-0145-y

The Mindfulness All-Party Parliamentary Group. (2015). Mindful nation $U K$. London (UK). Retrieved from http://themindfulnessi
nitiative.org.uk/images/reports/Mindfulness-APPG-Report_Mindf ul-Nation-UK_Oct2015.pdf.

Publisher's Note Springer Nature remains neutral with regard to jurisdictional claims in published maps and institutional affiliations. 